\title{
Picometer Scale Differences of Lattice Spacing In TEM Images
}

\author{
Martin Rose* and P.Fraundorf** \\ * Physics and Astronomy and Center for Molecular Electronics, University of Missouri - St.Louis, \\ St.Louis, MO 63121 USA
}

There are many applications in modern science in which a very precise knowledge of atom spacings i.e. lattice parameters is crucial. These include multi layer hetero structures in device manufacturing and core-rim nano particles, for example. In general it is interesting to know the change in spacings - or the strain - in the proximity of a lattice defect or a coherent interface between two different substances. From the knowledge of the spacings e.g. relaxation processes can be studied. Mathematical transforms intermediate between direct and reciprocal space make possible digital darkfield strategies for mapping strain relaxation around interfaces and dislocations [1-5]. Here we examine applications to the measurement of lattice parameter differences between adjacent phases.

A digitized photograph of a HRTEM image serves as input. The FFT is calculated to obtain the power spectrum (PS). One spot in the PS is chosen as a reference frequency, although the precise reference frequency (g-vector) will be chosen later. Next a smooth aperture centered around the chosen point is applied, and the data is shifted so that the reference point is at the DC peak. Calculating the inverse FFT of the shifted data yields a complex image, which can be interpreted as a darkfield (DF) image. Measurements can only be made at relatively bright areas in the DF image. The phase-gradient of the complex data parallel to the g-vector can be interpreted as a strain map. The actual value at each point in the strain map - referred to as $d$ - describes the deviation of the frequency (i.e. spacing) at this point in the image from the chosen reference frequency. Our algorithm minimizes $d$ by varying the g-vector, as the measurements on computer generated images were most precise when $d$ is minimal. The result of the measurement algorithm is the spacing that corresponds to the chosen base frequency multiplied by $1+\mathrm{d}$. It's a strength of this method that the result does not depend on the reference (i.e. g-vector) chosen by the user.

Fig. 1 shows the computer generated input where atoms are represented by Gaussian shaped peaks at positions marked by a 2D Bravais lattice [4]. As the arrangement of atoms is periodic straight lines can be drawn and parallels can be found. The distance between these parallels defines the spacing. The solid line in Fig. 1 marks the point, where the length of one base vector is multiplied by 1.012 which leads to a step in various spacings. At another position the base vector is multiplied again by 1.006. The upper right shows the PS and the g-vector of the spacing that was examined. At the upper left corner of the input the spacing is 5.3853 pixels. Fig. 2 shows the strain map which clearly shows the two steps in spacing, the three regions with constant spacing that appear flat in the strain map and the spacings determined by the algorithm. The error in the spacings measured is less than $1 \%$ !

Fig. 3 shows one of the two different regions of the same HRTEM image where measurements were made to show that variations in the spacing corresponding to the same g-vector can be measured accurately across the image. Fig. 4 illustrates the strain maps at the specific regions while the periodicity that is to be examined runs perpendicular to the g-vector. As the experimental data contains more noise the value of the strain map is averaged over an area of radius 100 pixels which reduces the spatial resolution of the method. A spacing of $6.14 \pm 0.01$ pixels was measured in the 
lower left area which is a $9 \pm 3 \%$ increase compared to $6.08 \pm 0.01$ pixels in the area to the right. Since the diffraction spot comes from a $\sim 2.6 \AA \mathrm{GaN}$ (002) spacing in an InGaN/GaN/AlGaN/GaN on sapphire heterostructure grown by Dan Leopold, the observation (neglecting gradient shifts due to other processes) corresponds to a projected lattice spacing difference of $2.3 \mathrm{pm}$ detected with an error of $1 \mathrm{pm}$.

\section{References}

[1] P. Fraundorf and G.K. Fraundorf, Proc 47th Ann. EMSA Meeting (1989) 122-123

[2] P. Fraundorf, Digital Darkfield Decompositions (arXiv:cond-mat/0403017)

[3] O.Saxton, Images of distorted crystals, The Newsletter of Synoptics Ltd, Spring 1990

[4] E.Snoeck et al, Thin Solid Films 319 (1998) 147-162

[5] C.L. Johnson, M.J. Hÿtch and P.R. Buseck, Amer. Min. 89 (2004) 1374-1379
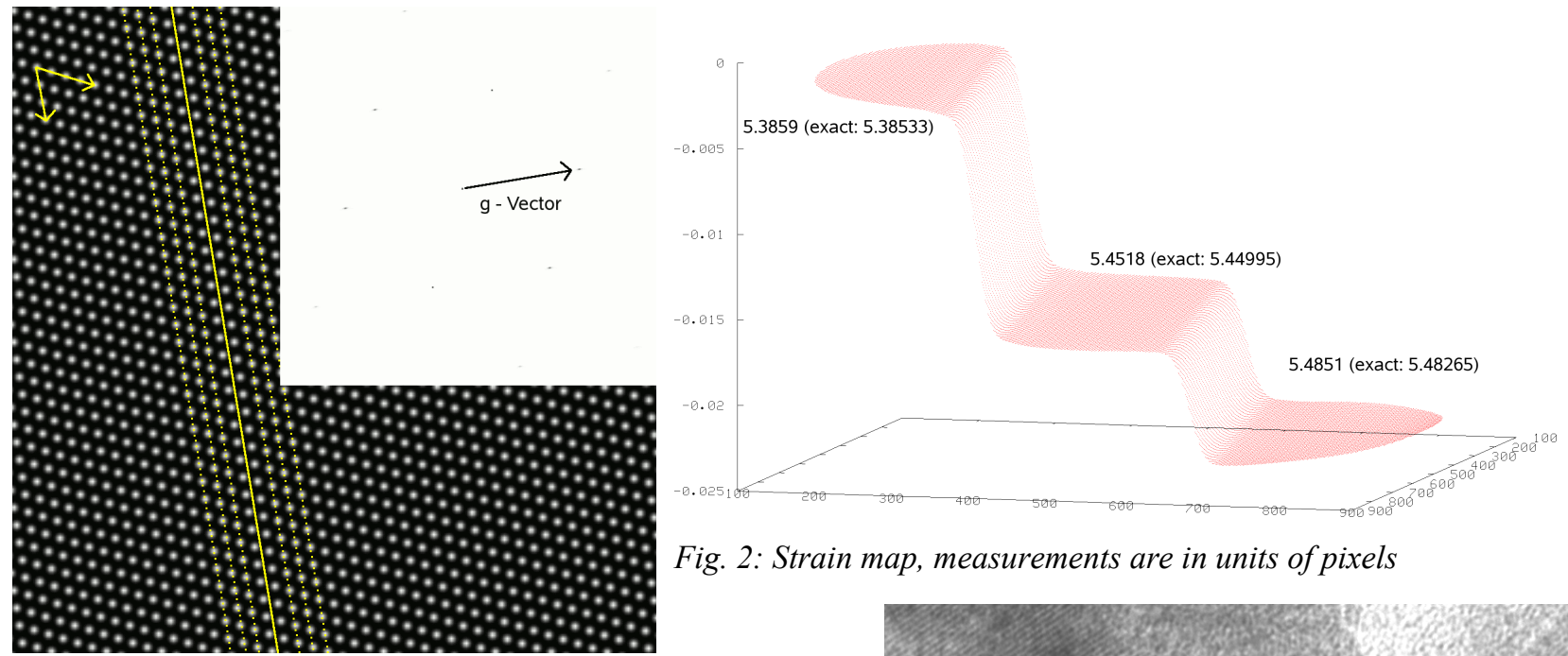

Fig. 2: Strain map, measurements are in units of pixels

Fig. 1: Computer generated lattice, periodicities perpendicular to the g-vector and PS
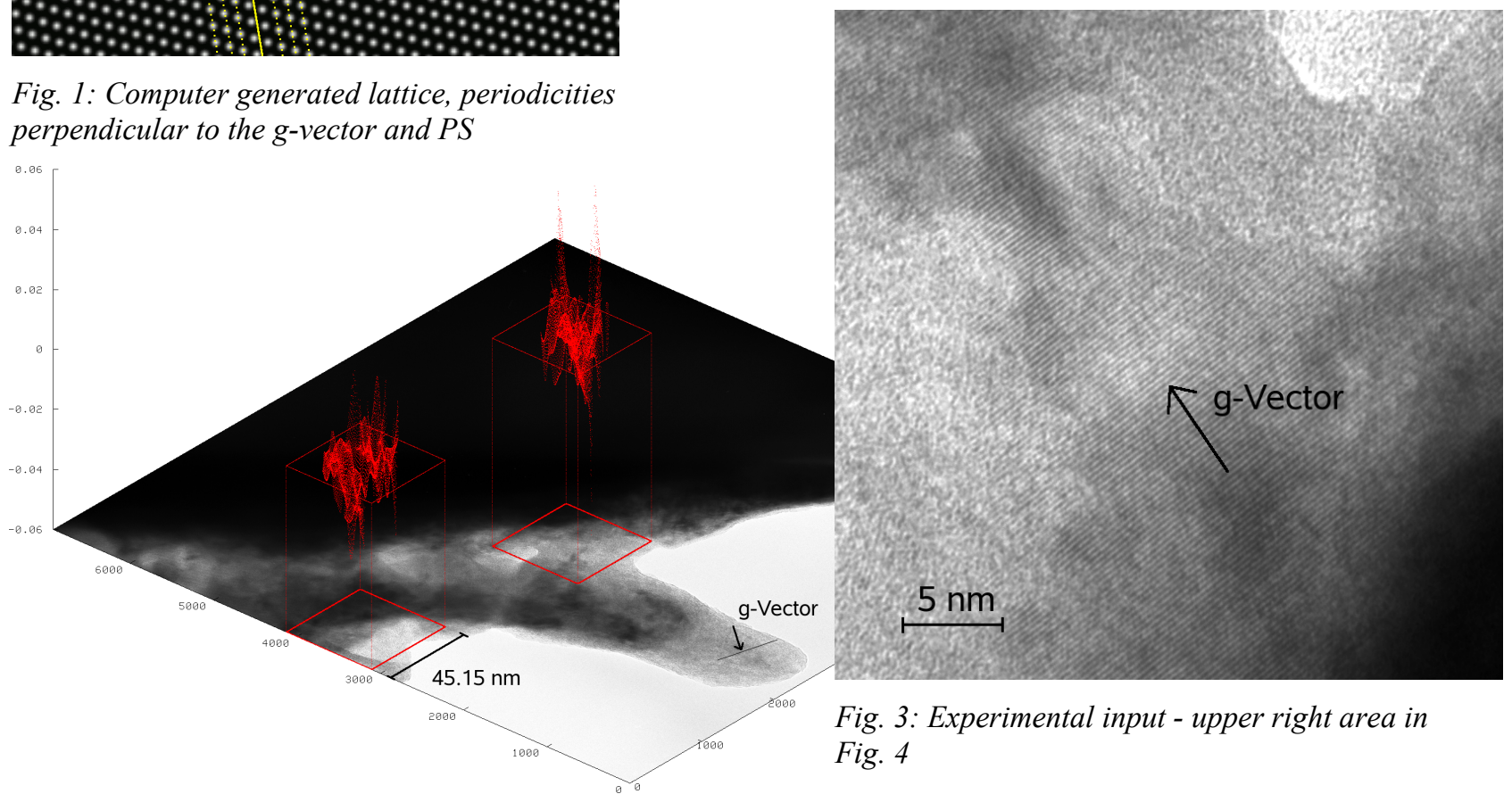

Fig. 3: Experimental input - upper right area in Fig. 4

Fig. 4: Comparison of spacing measurements in different regions of the image 